\title{
Enhanced Autoionization of Water at Phospholipid Interfaces
}

\author{
Alireza Mashaghi, ${ }^{* \dagger}$ P. Partovi-Azar, ${ }^{\ddagger}\|\|$ Tayebeh Jadidi, ${ }^{\ddagger}$ Mehrnaz Anvari, ${ }^{\S}$ Sara Panahian Jand, ${ }^{\ddagger}$ \\ Nasser Nafari, ${ }^{\Uparrow}$ M. Reza Rahimi Tabar, ${ }^{\S, \perp}$ Philipp Maass, ${ }^{\ddagger}$ Huib J. Bakker, ${ }^{\dagger}$ and Mischa Bonn ${ }^{\#}$ \\ ${ }^{\dagger}$ FOM Institute AMOLF, Science Park 104, 1098XG Amsterdam, The Netherlands \\ ${ }^{\ddagger}$ Fachbereich Physik, Universität Osnabrück, Barbarastraße 7, 49076 Osnabrück, Germany \\ ${ }^{\S}$ Department of Physics, Sharif University of Technology, 11365-9161 Tehran, Iran \\ "Institute for Research in Fundamental Sciences (IPM), P.O. Box 19395-5531, Tehran, Iran \\ ${ }^{\perp}$ Institute of Physics, Carl-von-Ossietzky University, D-26111 Oldenburg, Germany \\ \#Max Planck Institute for Polymer Research, Ackermannweg 10, 55128 Mainz, Germany \\ Supporting Information
}

\begin{abstract}
The structure and autoionization of water at the water-phospholipid interface are investigated by ab initio molecular dynamics and ab initio Monte Carlo simulations using local density approximation (LDA) and generalized gradient approximation (GGA) for the exchange-correlation energy functional. Depending on the lipid headgroup, strongly enhanced ionization is observed, leading to the dissociation of several water molecules into $\mathrm{H}^{+}$and $\mathrm{OH}^{-}$per lipid. The results can shed light on the phenomena of the high proton conductivity along membranes that has been reported experimentally.
\end{abstract}

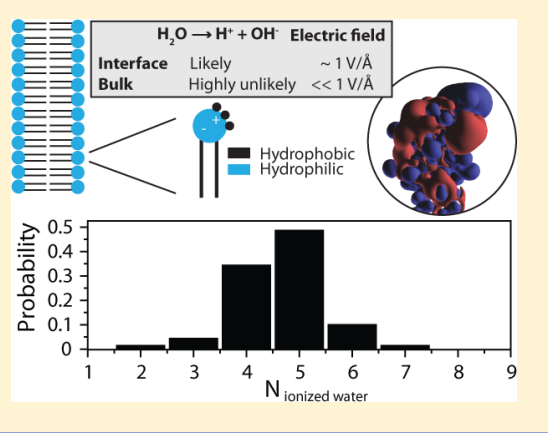

\section{INTRODUCTION}

Phospholipids are amphiphilic molecules that, in an aqueous environment, self-assemble into bilayers and form the major structural constituents of biomembranes. Although the affinity of the lipid's headgroup to interfacial water has been widely addressed in the literature, ${ }^{1,2}$ the exact chemical nature of this coupling is not fully understood and its strength remains to be quantified. In particular, it is not clear how this coupling affects the structure and dynamics of the interfacial water layer and the proton transport. Enhanced proton conduction along phospholipid-water interfaces was first observed in the mid 1980s. ${ }^{3,4}$ Recent studies by means of scanning tunneling microscopy (STM) confirm a significant lateral conductivity. This conductivity is believed to be of functional importance because lateral proton diffusion along membrane surfaces represents the most efficient pathway for $\mathrm{H}^{+}$transport between protein pumps. ${ }^{6}$ The molecular mechanism underlying the high lateral proton conductivity has not yet been resolved. ${ }^{7-11}$

In this paper we report ab initio molecular dynamics (AIMD) and $\mathrm{ab}$ initio Monte Carlo (AIMC) simulations of interfacial water covering the headgroup of zwitterionic dipalmitoyl-phosphatidylcholine (DPPC) molecule. We perform calculations within the local density approximation and generalized gradient approximation for the exchange-correlation energy functional, with Ceperley-Alder ${ }^{12}$ and PerdewBurke-Ernzerhof ${ }^{13}$ parametrizations, respectively. We show that the interfacial water exhibits a strongly enhanced autoionization that is caused by the presence of strong local electric field as well as strong hydrogen bonding.

\section{COMPUTATIONAL DETAILS}

Ground State Calculations. To calculate the ground state structure of the DPPC molecule, we first build the molecule by putting atoms together with coordinations according to the bonding rules from chemistry. To relax this structure, density functional theory (DFT) is applied as implemented in the SIESTA code. ${ }^{14}$ To this end, the DPPC molecule is placed in a unit cell with dimensions much larger than the size of the molecule. The spatial extension of the pure DPPC structure is $\approx 4 \times 29 \times 8 \AA$. We choose a box of $20 \times 40 \times 20 \AA$ dimensions. As a consequence, because we are dealing with a molecule (a cluster), no periodic boundary conditions are adopted and only the $\Gamma$ point in the reciprocal space is needed for the energy integration. The mesh cutoff is set to $450 \mathrm{Ry}$ constants, which is the smallest value above which the ground state energy as a function of the mesh-cutoff is found to become almost constant. This results in a fine real-space mesh with a mesh point at each $5 \times 10^{-4} \AA^{3}$. In the DFT calculations a double- $\zeta$ basis set is used along with one polarization orbital (DZP). This means that two confined orbitals are used for each magnetic quantum number $(m)$ in the valence shell of every chemical element. Polarization orbitals are added for a more precise description of the deformation of the atomic orbitals, if the atoms are part of the molecule. For example, for the carbon atoms (which are all part of the molecule), this choice results in 13 basis functions, 8 double- $\zeta$ functions, and 5 polarization

Received: December 5, 2012 
orbitals. We perform calculations within the local density approximation LDA and generalized gradient approximation GGA.

We check that the electronic and structural properties (for instance, lipid length, tilt angle of the tail, charge density profile, electrostatic potential, etc.) for a given molecule conformation are almost the same with different choices for exchangecorrelation functionals including local spin density approximation (LSDA) and generalized gradient approximation (GGA). For the relaxation of the molecular structure the conjugate gradient scheme is applied. When the maximum difference between the output and the input on each element of the density matrix in a self-consistent cycle is smaller than $10^{-5}$, the charge density is considered to have converged. To avoid local minima in the energy landscape, we set the maximum displacement of the atoms to a large value of 0.5 of the Bohr radius in the conjugate gradient scheme. The molecule is considered to be in the relaxed state when the maximal force on the atoms falls below $0.005 \mathrm{eV} / \AA$.

To find the ground state of the hydrated lipid, we first obtain the relaxed structure of a single water molecule within the DFT scheme described above. We then place 20,50 , or 75 replicas of water molecules around the relaxed structure of the DPPC molecule in such a way that the water molecules cover the hydrophilic headgroup. The DPPC $/\left(\mathrm{H}_{2} \mathrm{O}\right)_{20}, \mathrm{DPPC} /\left(\mathrm{H}_{2} \mathrm{O}\right)_{50}$, and DPPC $/\left(\mathrm{H}_{2} \mathrm{O}\right)_{75}$ complexes are then relaxed again with the protocol explained above. In the case of hydrated samples, we use $30 \times 50 \times 30,30 \times 60 \times 30$, and $30 \times 70 \times 30$ simulation boxes for DPPC/ $\left(\mathrm{H}_{2} \mathrm{O}\right)_{20}$, DPPC $/\left(\mathrm{H}_{2} \mathrm{O}\right)_{50}$, and DPPC/ $\left(\mathrm{H}_{2} \mathrm{O}\right)_{75}$, respectively, with no periodic boundary conditions.

The electronic properties and vibrational eigenmodes of the above structures, i.e., pure and hydrated DPPC samples, are reported in our two most recent works ${ }^{15,16}$ Generally speaking, obtaining a negative (imaginary, because what is obtained is actually $\omega^{2}$ rather the $\omega$ ) frequencies is a signature of having an unstable structure that is energetically very sensitive to small changes in atomic positions. It has been shown that apart from three, and only three, frequencies with $|\omega|<0.1 \mathrm{~cm}^{-1}$ (corresponding to three acoustic modes), all the other eigenfrequencies (total 390 eigenfrequencies for pure, 570 for DPPC $/\left(\mathrm{H}_{2} \mathrm{O}\right)_{20}$, and 840 for DPPC $\left./\left(\mathrm{H}_{2} \mathrm{O}\right)_{50}\right)$ are positive.

To get insight into the ground state structure of a lipid monolayer, we also build a hydrated 2-DPPC $/\left(\mathrm{H}_{2} \mathrm{O}\right)_{64}$ complex, where the two relaxed DPPCs are in parallel with opposite dipole moment vectors. The relaxed configuration obtained from this arrangement resembles the structural unit of a membrane monolayer. ${ }^{15}$ In this case, we use periodic boundary conditions, with a $3 \times 3 \times 1$ Brillouin-zone sampling. We also let the unit cell be optimized at the same time with the atoms with the maximum stress tolerance set to $0.25 \mathrm{GPa}$.

Inclusion of van der Waals (vdW) interactions is generally thought to be necessary for molecules with hydrogen bonds. We hence perform simulations also with the vdW functional suggested in refs 17 and 18 for the pure and hydrated DPPC. The structures and electronic properties turn out to be quite independent of the inclusion of the vdW functional. ${ }^{15}$ For example, the difference between the length of the lipid in vdW calculation (28.30 ̊) and the reported LDA calculation (28.24 $\AA$ ) is less than $0.3 \%$ (please see ref 15 ).

Ab Initio Molecular Dynamics Simulations. Ab initio molecular dynamics (AIMD) within LDA simulations are performed with a time step $\Delta t=0.1 \mathrm{fs}$, which is about one hundredth of the shortest vibration time (inverse vibrational frequency) in the system. Nosé mass, which controls the coupling of the thermal bath to the system, is set to $100.0 \mathrm{Ry}$ $\mathrm{fs}^{2}$. In these simulations the forces acting on the atoms are calculated from the charge density obtained from the DFT. The simulation is carried out for a period of 3 ps (30 000 time steps; 10 month CPU time on 12 parallel processors, Intel(R) Xeon(R) CPU, X5650 @ $2.67 \mathrm{GHz}$ ) in an NVT ensemble for the (isolated) DPPC $/\left(\mathrm{H}_{2} \mathrm{O}\right)_{50}$ complex (no periodic continuation). The temperature is kept fixed at $300 \mathrm{~K}$ with the NoséHoover thermostat. Temperature as a function of time is shown in Figure S1 (Supporting Information). The thermal equilibrium is reached within the first 0.2 fs.

Ab Initio Monte Carlo Simulations. We note that, there can be large energy barriers, which can lead to molecules getting trapped in a few low energy conformations in an AIMD simulation, leading to poor conformational sampling. In contrast, the random moves in an AIMC simulation can easily lead to barrier crossings. The standard Metropolis algorithm is used in our ab initio MC simulations. The trial conformations at each MC step, obtained by randomly displacing the atoms, are sent to SIESTA, as an input, and their total energy is sent back by SIESTA to our code. SIESTA is treated as a subroutine. We choose the distance for the maximum movement of $\mathrm{H}$ atoms $\left(d_{\mathrm{H}}\right)$ in such a way that the acceptance ratio of MC steps in AIMC within GGA and LDA is in the interval $30-40 \%$. The maximum movement of the other atoms $(\mathrm{x})$ is controlled by their mass, i.e., $d_{\mathrm{x}}=\left(m_{\mathrm{H}} / m_{\mathrm{x}}\right) d_{\mathrm{H}}$, and we fix the temperature to be $300 \mathrm{~K}$. The simulation evolves according to the following steps: (i) Given a configuration $\mu$ and its energy $E_{\mu}$, a new configuration $\nu$ is generated by random displacements of all particles simultaneously. All configuration energies are obtained from an ab initio calculation, as explained above within LDA and GGA. (ii) The energy of the new configuration $\left(E_{\nu}\right)$ is then obtained, and if $\Delta E=E_{\nu}-E_{\mu}<0$, we accept the new coordinates. Otherwise, if $\Delta E \geq 0$ we select a random number $R$ in the range $[0,1]$ and if $\exp (-\beta \Delta E)>R$ we accept the new coordinates and if $\exp (-\beta \Delta E) \leq R$, we reject the movement. Here $\beta=1 / k_{\mathrm{B}} T$, where $T$ and $k_{\mathrm{B}}$ are temperature and the Boltzmann constant, respectively. (iii) If the configuration $\nu$ is rejected, the old one is maintained, and if accepted, it serves as the configuration in the next step. Figure S2 (Supporting Information) shows the total energy versus MC steps. Although the fluctuations of the total energy are in the range of $4 \mathrm{meV}$, the averages for calculating the structural properties are taken over the 3000 sampling configurations after equilibration. The fact that the total energy fluctuates around the energy of the initial configuration, and it does not tend to lower values (better minima), shows that the global minimum is reached through the conjugate gradient optimization.

\section{RESULTS AND DISCUSSION}

The ground state (i.e., zero temperature) of DPPC with 50 water molecules hydrating its headgroup is obtained using density functional theory (DFT) as implemented in the SIESTA code. $^{14,16}$ The calculated ground state structure is used as the starting configuration for AIMD and AIMC simulations at $300 \mathrm{~K}$ (Figure 1a). The AIMD simulations are performed $^{14,19,20}$ up to a time of 3 ps with a time step $\Delta t=0.1$ $\mathrm{fs}$, which is about one hundredth of the shortest vibration period in this system. ${ }^{16}$ The temperature is kept fixed with a Nosé-Hoover thermostat. ${ }^{21,22}$

In bulk liquid water, autoionization is a very unlikely process with a probability of $1.8 \times 10^{-9}$ at $300 \mathrm{~K}$. At the DPPC-water 
a

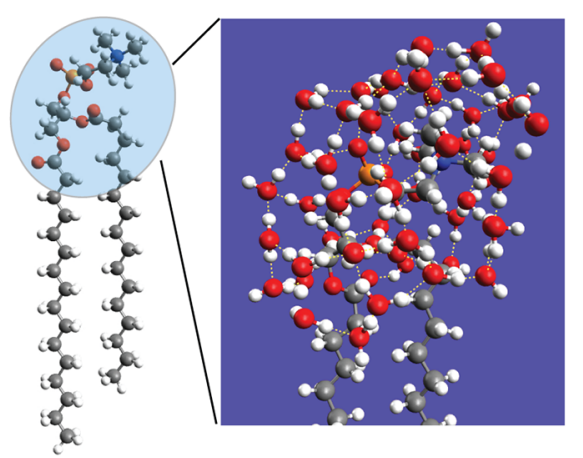

b

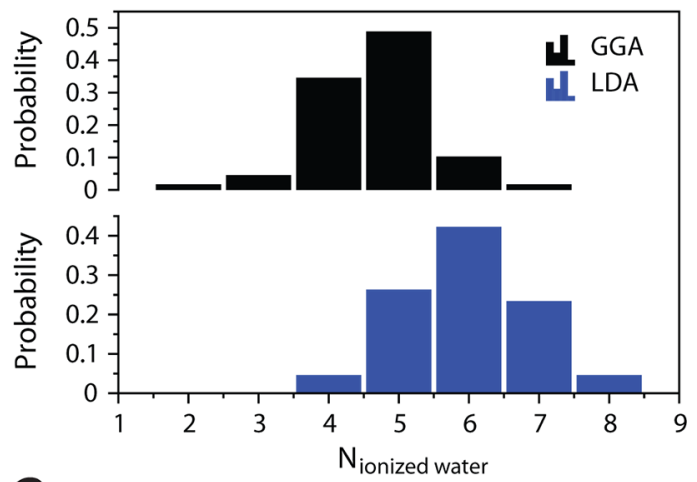

C

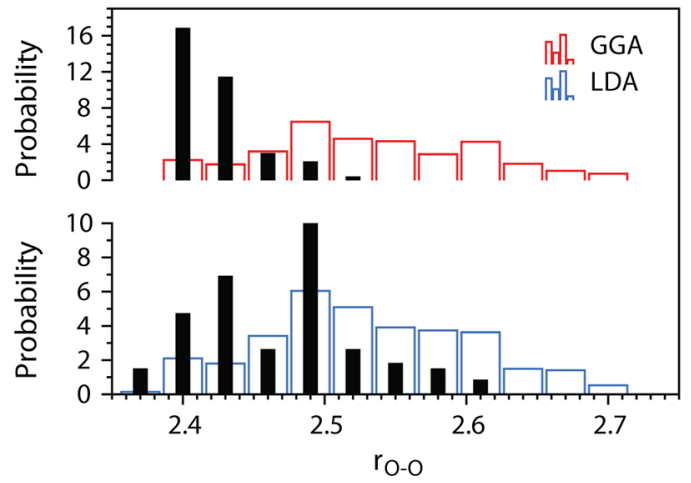

Figure 1. (a) Ground state structure of $\mathrm{DPPC} /\left(\mathrm{H}_{2} \mathrm{O}\right)_{50}$. (b) Probability of water ionization at $300 \mathrm{~K}$, estimated by sampling configurations from the AIMC simulation within LDA and GGA. (c) Probability density of $r(\mathrm{O}-\mathrm{O})$ distances of neighboring water molecules in AIMC for DPPC $/\left(\mathrm{H}_{2} \mathrm{O}\right)_{50}$ within LDA and GGA at $T$ $=300 \mathrm{~K}$. The area under the histogram in an interval gives the probability of $r(\mathrm{O}-\mathrm{O})$ to be in that interval. The probability densities of $r(\mathrm{O}-\mathrm{O})$ distances of neighboring water molecules involved in proton-transfer processes are shown with black bars. Water molecules prone to autodissociation in the subsequent AIMC simulations within LDA and GGA at $300 \mathrm{~K}$ are found to show $2.37<r(\mathrm{O}-\mathrm{O})<2.61 \AA$ and $2.40<r(\mathrm{O}-\mathrm{O})<2.52 \AA$, respectively.

interface, we observe an enhanced ionization of water even in the ground state configuration, using both local density approximation and generalized gradient approximation. In the ground state of the DPPC $/\left(\mathrm{H}_{2} \mathrm{O}\right)_{50}$ complex we find two water molecules dissociated into $\mathrm{H}^{+}$and $\mathrm{OH}^{-}$, with the $\mathrm{OH}^{-}$ions located in the vicinity of the choline group $\left[-\left(\mathrm{CH}_{2}\right)_{2}-\right.$ $\left.\mathrm{N}\left(\mathrm{CH}_{3}\right)_{3}\right]^{+}$. Upon removal of the DPPC molecule, the water cluster rearranges and the $\mathrm{H}^{+}$and $\mathrm{OH}^{-}$ions reassociate. When the number of water molecules around the headgroup is increased to 75 , the $\mathrm{H}^{+}$number does not increase, indicating that autoionization is catalyzed by the interface and is specific to the interface. Reducing the number of water molecules to less than 20 leads to disappearance of the autoionization. For a system consisting of two neighboring hydrated DPPC molecules, we find again that two water molecules per lipid molecule become autoionized in the ground state configurations. At finite temperature, the number of ionized water molecules fluctuates in time in AIMD and AIMC steps. Figure $1 \mathrm{~b}$ shows the probability of finding $N_{\mathrm{H}^{+}}$ionized water molecules in the system at $300 \mathrm{~K}$, as obtained from the AIMC simulations within LDA and GGA for DPPC/ $\left(\mathrm{H}_{2} \mathrm{O}\right)_{50}$. We find $\left\langle N_{\mathrm{H}^{+}}\right\rangle_{\mathrm{LDA}}=$ $6.0 \pm 0.9$ and $\left\langle N_{\mathrm{H}^{+}}\right\rangle_{\mathrm{GGA}}=4.7 \pm 0.8$, respectively, i.e., a further increase of autodissociation compared to the that in ground state by a factor of about 3. In the AIMD simulations we find the average number of ionized water molecules to be $\left\langle N_{\mathrm{H}^{+}}\right\rangle_{\mathrm{AIMD}}$ $=4.6 \pm 1.6$. The $\mathrm{O}-\mathrm{O}$ distances distribution of the neighboring water molecules in AIMC simulation within LDA and GGA are shown in Figure 1c. The averaged $\mathrm{O}-\mathrm{O}$ distance of neighboring water molecules are about 2.50 and $2.55 \AA$ from AIMC simulations within LDA and GGA, respectively. ${ }^{23-25}$

The origin of the enhanced autoionization lies in the presence of strong local electric field as well as strong hydrogen bonding. ${ }^{26,27}$ The interfacial water is subject to a strong electric field of the lipid, ${ }^{15}$ which originates primarily from the large dipole moment associated with the negatively charged phosphate and the positively charged choline groups of the DPPC molecule. The dipole moment is $\sim 10 \mathrm{D}$, several fold larger than the dipole moment of a water molecule. ${ }^{15,28}$ Electric field-induced enhancement of water dissociation has been experimentally observed previously using field emitter tips where the surface electric fields are estimated to be on the order of $1 \mathrm{~V} / \AA^{27}$ Fields of this magnitude distort molecules, induce ionization and break bonds, and thus lead to an increased dissociation constant of interfacial water. In Figure 2, the

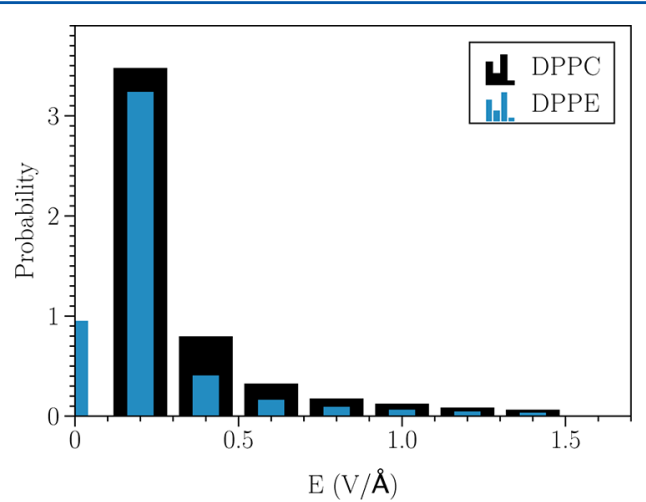

Figure 2. Probability distribution of the magnitude of the electric fields experienced by water molecules hydrating DPPC and DPPE molecules. The threshold value to ionize water molecules is estimated to be $\sim 1 \mathrm{~V} / \AA^{27}{ }^{27}$ The electric field distribution was calculated from a spherical region (radius $=1 \AA$ ) at $3 \AA$ distance (the Lennard-Jones radius of $\mathrm{O}$-atom in water molecule) from the water layer along the long axis of the molecule.

probability density function of the magnitude of the electric field around the water molecules of hydrated DPPC molecule is given. Clearly, there are many points in the space around the water molecules, where the local electric field strength exceeds $1 \mathrm{~V} / \AA$. Also in Figure 3 we plot the isosurfaces for the potentials $\pm 500 \mathrm{mV}$ around the hydrated DPPC, which shows 
strong fluctuations of potential about $\Delta V \simeq 1 \mathrm{~V}$ on a $\sim 1 \AA$ length scale.

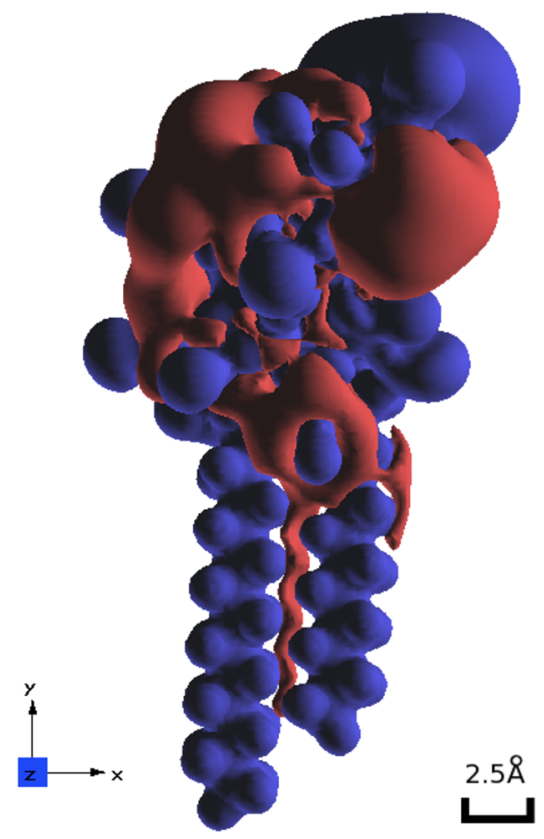

Figure 3. Potential isosurfaces of DPPC $/\left(\mathrm{H}_{2} \mathrm{O}\right)_{50}$ for $V=500 \mathrm{mV}$ (red) and $V=-500 \mathrm{mV}$ (blue) in the ground state. Strong spatial variations of the potential about $\Delta V \simeq \pm 1 \mathrm{~V}$ on a length scale $\sim 1 \AA$ are clearly seen.

We note, as shown in Figure 1c, that water molecules that get involved in proton-transfer processes have $\mathrm{O}-\mathrm{O}$ distances of about $2.37<r(\mathrm{O}-\mathrm{O})<2.61 \AA$ and $2.40<r(\mathrm{O}-\mathrm{O})<2.52 \AA$, for AIMC simulations within LDA and GGA, respectively. In bulk liquid water, where water autoionization is a rare event, the $\langle r(\mathrm{O}-\mathrm{O})\rangle$ distance has been calculated and measured to be larger than the values observed at the lipid interface: reported values for the $r(\mathrm{O}-\mathrm{O})$ distance vary depending on the techniques used (2.78-2.82 $\AA$ from neutron and X-ray diffraction studies; 2.5-3.5 $\AA$ from EXAFS). ${ }^{29-32}$ With decreasing $\mathrm{O}-\mathrm{O}$ distance, the hopping matrix element of $\mathrm{H}$ transfer increases, facilitating proton hopping. Similarly, association of $\mathrm{H}^{+}$and $\mathrm{OH}^{-}$ions in water also proceeds via hydrogen-bond contraction and involves a collective compression of the water-wire bridging the ions. ${ }^{33}$ We note that the observed distances in our simulations is in agreement with experimental findings 34,35

To demonstrate that the observation of autoionization is not an artifact of our approach, we determine the structure of water molecules hydrating the zwitterionic phosphatidylethanolamines (PE). No autoionization is observed in the ground state configuration within GGA. This result can be understood by inspection of Figure 2: the number of water molecules around DPPE that experience a local electric field exceeding 1 $\mathrm{V} / \AA$ is lower than for the hydrated DPPC. The probability densities of $r(\mathrm{O}-\mathrm{O})$ distances of neighboring water molecules for DPPC $/\left(\mathrm{H}_{2} \mathrm{O}\right)_{50}$ and DPPE $/\left(\mathrm{H}_{2} \mathrm{O}\right)_{50}$ within GGA in ground state configuration given in Figure 4 are consistent with this picture. For DPPE $/\left(\mathrm{H}_{2} \mathrm{O}\right)_{50} r(\mathrm{O}-\mathrm{O})$ distances less than $2.5 \AA$ are rare, and autoionization within the DPPE/ $\left(\mathrm{H}_{2} \mathrm{O}\right)_{50}$ cluster is thus unlikely. This behavior can in part be traced back to the electric dipole moment of DPPE being about $\sim 8.5 \mathrm{D}$, which is $\sim 15 \%$ smaller than that of the DPPC

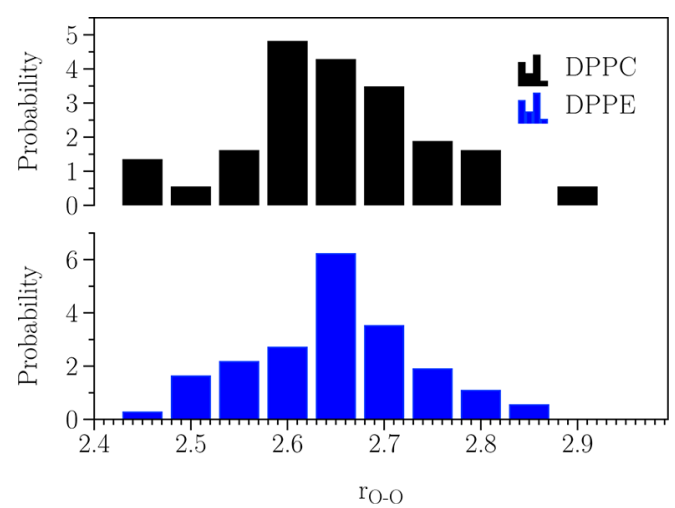

Figure 4. Probability distribution of $r(\mathrm{O}-\mathrm{O})$ distances of neighboring water molecules for DPPC $/\left(\mathrm{H}_{2} \mathrm{O}\right)_{50}$ and DPPE $/\left(\mathrm{H}_{2} \mathrm{O}\right)_{50}$ within GGA in their ground state configuration. For DPPE $/\left(\mathrm{H}_{2} \mathrm{O}\right)_{50} r(\mathrm{O}-\mathrm{O})$ always exceeds $2.5 \AA$, consistent with the absence of autoionized water molecules. In contrast, for DPPC $/\left(\mathrm{H}_{2} \mathrm{O}\right)_{50}$, two autoionized water molecules are found in the ground state configuration.

molecule. ${ }^{15}$ We observed a reduction in the spread of the $\langle r(\mathrm{O}-\mathrm{O})\rangle$ at the DPPE interface of nearly $12.5 \%$, indicating a stronger homogeneity of the electric field. Moreover, hydrophobic character of the three methyl groups in DPPC, which are absent in DPPE, may also contribute to the higher probability of autoionization and proton-transfer rate at DPPC interfaces. ${ }^{36}$ We note that created hydroxide ions in the DPPC/ water system prefer to localize at the interface region close to the hydrophobic methyl groups, a phenomenon observed at other hydrophobic interfaces as well. ${ }^{36,37}$

We note that enhanced water autoionization cannot be explained from an increase in the strength of the hydrogenbond interaction. Both DPPC and DPPE are strongly interacting with the interfacial water molecules, with DPPE making even more $\mathrm{H}$-bonds with the water layer. The hydrogen-bond interaction, however, does play a role in keeping the water molecules in close proximity of the lipid headgroup and thereby exposing them to the strong electric field exerted by the lipid.

\section{SUMMARY AND OUTLOOK}

In summary, we observe that water molecules display strongly enhanced autoionization at the lipid interface, as a result of the strong local electric field and the resulting reduced $\mathrm{O}-\mathrm{O}$ distances of the water molecules located around the PC headgroup. The observation of enhanced autodissociation is expected to be relevant to the properties of water at a membrane interface. The strongly enhanced autodissociation reported here results in a significant local charge flux within the interfacial water layers and may explain the very high lateral conductivity associated with proton mobility observed for PC membranes. The enhanced ionization of water and concomitant charge mobility at PC lipids is highly relevant to biology, because phosphatidylcholines (PC) are the most common lipids in the outer leaflet of cell membranes. This study is a step toward understanding the water ionization process at the lipid interface using first principle methods. Further analysis is needed to quantify how the ionized water molecules indeed contribute to electrical conductivity of the system. 


\section{ASSOCIATED CONTENT}

\section{S Supporting Information}

Supplemental figures illustrating temperature (S1) and total energy (S2) as a function of time and Monte Carlo steps respectively. This material is available free of charge via the Internet at http://pubs.acs.org.

\section{AUTHOR INFORMATION}

\section{Corresponding Author}

*E-mail: mashaghi@amolf.nl.

Notes

The authors declare no competing financial interest.

\section{ACKNOWLEDGMENTS}

We are grateful to Davide Donadio and Yuki Nagata for their valuable comments. M.R.R.T. thanks the Deutsche Forschungsgemeinschaft (DFG grant No. 190/135-1) for financial support. A.M. and H.J.B. are supported by the research program of the Foundation for Fundamental Research on Matter (FOM), which is part of The Netherlands Organization for Scientific Research (NWO).

\section{REFERENCES}

(1) Pasenkiewicz-Gierula, M.; Takaoka, Y.; Miyagawa, H.; Kitamura, K.; Kusumi, A. J. Phys. Chem. A 1997, 101, 3677-3691.

(2) Foglia, F.; Lawrence, M.; Lorenz, C.; McLain, S. J. Chem. Phys. 2010, 133, 145103.

(3) Teissié, J.; Prats, M.; Soucaille, P.; Tocanne, J. F. Proc. Natl. Acad. Sci. 1985, 82, 3217-3221.

(4) Prats, M.; Teissié, J.; Toccane, J. F. Nature 1986, 322, 756-758.

(5) Heim, M.; Cevc, G.; Guckenberger, R.; Knapp, H. F.; Wiegrabe,

W. Biophys. J. 1995, 69, 489-497.

(6) Serowy, S.; Saparov, S. M.; Antonenko, Y. N.; Kozlovsky, W.; Hagen, V.; Pohl, P. Biophys. J. 2003, 84, 1031-1037.

(7) Mulkidjanian, A. Y.; Heberle, J.; Cherepanov, D. A. Biochim. Biophys. Acta - Bioenerg. 2006, 1757, 913-930.

(8) Smondyrev, A. M.; Voth, G. A. Biophys. J. 2002, 82, 1460.

(9) Yamashita, T.; Voth, G. A. J. Phys. Chem. B 2010, 114, 592.

(10) Springer, A.; Hagen, V.; Cherepanov, D. A.; Antonenko, Y. N.; Pohl, P. Proc. Natl. Acad. Sci. U. S. A. 2011, 108, 14461.

(11) Agmon, N.; Gutman, M. Nat. Chem. 2011, 3, 840.

(12) Ceperley, D. M.; Alder, B. J. Phys. Rev. Lett. 1980, 45, 566.

(13) Perdew, J. P.; Burke, K.; Ernzerhof, M. Phys. Rev. Lett. 1996, 77, 3865.

(14) Soler, J. M.; Artacho, E.; Gale, J. D.; García, A.; Junquera, J.; Ordejón, P.; Sánchez-Portal, D. J. Phys.: Condens. Matter 2002, 14, 2745.

(15) Mashaghi, A.; Partovi-Azar, P.; Jadidi, T.; Nafari, N.; Maass, P.; Tabar, M. R. R.; Bonn, M.; Bakker, H. J. J. Chem. Phys. 2012, 136, 114709.

(16) Mashaghi, A.; Partovi-Azar, P.; Jadidi, T.; Nafari, N.; Esfarjani, K.; Maass, P.; Tabar, M. R. R.; Bakker, H. J.; Bonn, M. J. Phys. Chem. B 2012, 116, 6455.

(17) M. Dion, M.; E. S., D. L.; H. Rydberg, H.; Lundqvist, B. Phys. Rev. Lett. 2004, 92, 246401.

(18) G., G. R.-P.; Soler, J. Phys. Rev. Lett. 2009, 103, 096102.

(19) Anglada, E.; Junquera, J.; Soler, J. M. Phys. Rev. E 2003, 68, 055701.

(20) Hutter, J.; Marx, D. Ab Initio Molecular Dynamics Basic Theory and Advanced Methods; Cambridge University Press: Cambridge, U.K., 2009.

(21) Nosé, S. J. Chem. Phys. 1984, 81, 511.

(22) Hoover, W. G. Phys. Rev. A 1985, 31, 1695-1697.

(23) Laasonen, K.; Csajka, F.; Parrinello, M. Chem. Phys. Lett. 1992, 194, 172.
(24) Laasonen, K.; Parrinello, M.; Car, R.; Lee, C.; Vanderbilt, D. Chem. Phys. Lett. 1993, 207, 208.

(25) Rao, L.; Ke, H.; Fu, G.; Xu, X.; Yan, Y. J. Chem. Theory. Comput. 2009, 5, 86.

(26) Geissler, P. L.; Dellago, C.; Chandler, D.; Hutter, J.; Parrinello, M. Science 2001, 291, 2121-2124.

(27) Stuve, E. M. Chem. Phys. Lett. 2012, 519.

(28) Silvestrelli, P.; Parrinello, M. Phys. Rev. Lett. 1999, 82, 33083311.

(29) Xu, X.; Goddard, W. A. J. Phys. Chem. A 2004, 108, 2305.

(30) Bergmann, U.; Cicco, A. D.; Werner, P.; Principi, E.; Glatzel, P.; Nilsson, A. J. Chem. Phys. 2007, 127, 174504.

(31) Sharp, K. A.; Vanderkooi, J. M. Acc. Chem. Res. 2010, 43, 231.

(32) Soper, A. K. J. Phys.: Condens. Matter 2007, 19, 335206.

(33) Hassanali, A.; Prakash, M.; Eshet, H.; Parrinello, M. Proc. Natl. Acad. Sci 2011, 108, 20410-20415.

(34) Tielrooij, K.; Paparo, D.; Piatkowski, L.; Bakker, H.; Bonn, M. J. Chem. Phys. 2009, 97, 2484-2492.

(35) Roberts, S.; Ramasesha, K.; Tokmakoff, A. J. Chem. Phys. 2009, 42, 1239-1249.

(36) Zhang, C.; Knyazev, D.; Vereshaga, Y.; Ippoliti, E.; Nguyen, T.; Carloni, P.; Pohl, P. Proc. Natl. Acad. Sci. 2012, 109, 9744-9749.

(37) Car, R.; Kudin, K. N. J. Am. Chem. Soc. 2008, 130, 3915-3919. 